\title{
Performance Evaluation System of Cross-border B2B E-commerce Website
}

\author{
Lu-lian ZOU, Wen-zhuo ZHANG and Rui DONG
}

No.10 Xitucheng Road, HaiDian District, Beijing, China

\begin{abstract}
Keywords: Cross-border B2B E-commerce, E-commerce website, Performance evaluation, Fuzzy comprehensive evaluation, AHP.
\end{abstract}

\begin{abstract}
Under the environment of globalization and the increasingly vigorous development of e-commerce, B2B cross-border e-commerce websites has increasingly occupied a prominent position in the industry, and the study of evaluation system of B2B cross-border e-commerce websites has triggered a discussion. This paper studies the performance evaluation method of $\mathrm{B} 2 \mathrm{~B}$ cross-border e-commerce website. The study based on the AHP model, fuzzy comprehensive evaluation model and the improved model according to the characteristics of the research object. This paper constructs a performance evaluation system of B2B cross-border e-commerce website with 15 basic indicators by means of questionnaire investigation, expert evaluating and the combination of qualitative analysis and quantitative analysis. Furthermore, this paper aims to provide optimal suggestions for the development of websites and provide strategic guidance for policy makers through the research on the performance evaluation of such websites.
\end{abstract}

\section{跨境B2B电子商务网站绩效评价体系}

\author{
邹璐濂, 张文卓, 董睿 \\ 北京邮电大学，北京市，中国
}

关键词：跨境B2B电子商务; 电子商务网站; 绩效评价; 模糊综合评价; 层次分析法

摘要：在全球化和电子商务发展日益蓬勃的大环境下, B2B跨境电子商务网站在行业的地位 日益凸显, B2B跨境电子商务网站的评价体系研究成为了多方关注热点, 本文对B2B跨境电子 商务网站的绩效评价方法进行了研究。本文基于AHP模型、模糊综合评价模型, 并在一定基 础上结合研究对象的特点改进了模型, 使用了用问卷调查, 专家打分、定量分析结合定性分 析的方法, 构建了具有 15 个基础指标的B2B跨境电子商务网站绩效评价体系, 旨在通过对该 类网站的绩效评价研究, 给网站的发展提供优化建议, 给决策者提供战略指导。

\section{1. 引言}

20世纪90年代末互联网风潮到来, “外贸+互联网”模式出现, 许多企业开始依托互联网技 术进行跨国贸易。随着行业制度和相关法律法规的完善, 相关网站也逐渐走上规范化的道路, 人们也开始关注如何去评价一个电子商务网站。从一个网站的经营管理角度, 网站绩效评价 是一个不可忽视的方面。绩效是企业在一定的资源、条件和环境下, 完成任务的出色程度, 是对目标实现程度及达成效率的衡量与反馈, 作为一种重要的学习和反馈工具, 网站绩效评 价有利于企业的规范化发展，将网站的战略目标融入企业日常经营过程中。

本文通过构建B2B跨境电子商务网站绩效评价模型, 对该类网站绩效情况进行分析, 并 根据分析结果提出优化建议。 


\section{AHP模型与评价指标体系构建}

本文总体借鉴AHP层及分析法的思想与综合分析评价法相结合的思想，同时在逐层分析 中，采取模糊综合评价法并结合定量、定性方法具体分析评价。

首先对问题进行分解，构建出一个层次化结构体系：第一层“目标层”：B2B跨境电子商 务网站绩效；第二层“判断层”：分为“资金与财务”“社会与市场”“服务与管理”; 第三层“措施 层”: 根据指标的可获取性, 对上一层进一步分解细化为 15 个部分, 各层级的分析方法及指标 权重具体如下（图1）:

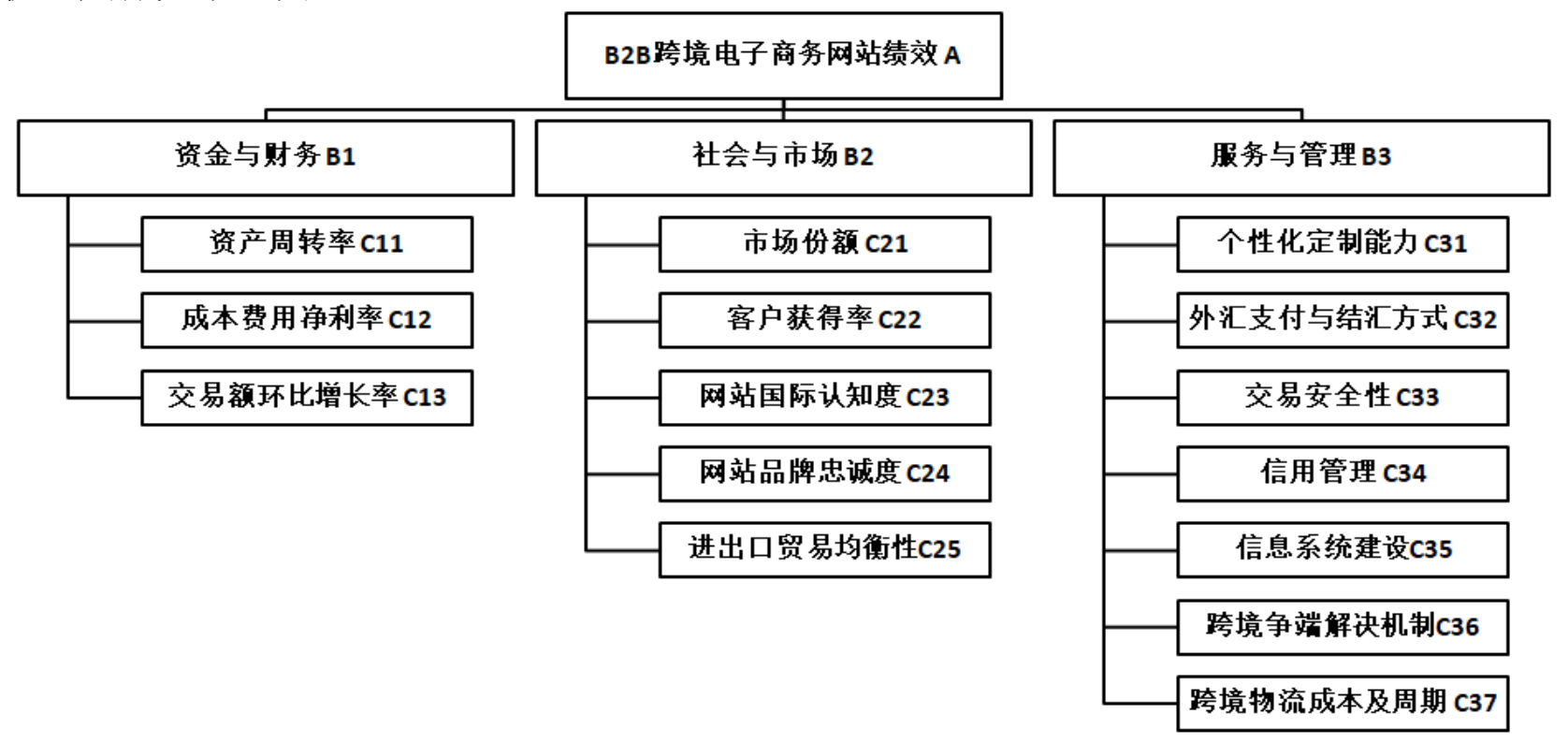

图1 指标层次图

\section{1 资金与财务}

本文选择三个方面的财务指标，鉴于财务数据具有易获取和易统计分析的特点，所以该 部分主要采用定量分析的方法。指标属性均为客观，数据来源为公司财务报表信息。

2.1.1 资产周转率

计算方法：资产周转率=主营业务收入/资产平均余额，其中：资产平均余额 $=($ 初期总资 产+期末总资产) $/ 2$

资产周转率是网站一定时期的主营业务收入与资产总额的比率，是反应资金流转速度指 标。在保证生产经营正常进行的情况下，资金周转速度越快，说明该网站资金利用效果越好。 2.1.2 成本费用净利率

计算方法：成本费用净利率＝净利润/成本费用总额×100\%

它反映企业生产经营过程中发生的耗费与获得的收益之间的关系。这一比率越高, 说明 企业为获得收益而付出的代价越小, 企业的盈利能力越强。因此, 通过这个比率不仅可以评 价企业盈利能力的高低，也可以评价企业对成本费用的控制能力和经营管理水平。

\subsection{3 交易额环比增长率}

计算方法：（本期交易额的值-上一期交易额值）/上一期交易额

该指标能够反映出网站的成长和发展能力, 是衡量网站经营状况预测网站经营业务拓展 趋势的重要指标, 也是网站扩张增量资本和存量资本的重要前提。该指标越大，表明其增长 速度越快, 该网站的发展前景越好。

\section{2 社会与市场}

\subsection{1 市场份额}

客观指标。数据来源: 市场调研机构。 
网站的销售量（额）在其目标市场, 即它所服务的市场中所占的比重。市场份额具有两 个方面的特性: 数量和质量, 它在很大程度上反映了该网站竞争地位和盈利能力。

2.2.2 客户获得率

客观指标。计算方式: 客户数量增长率=本期客户数量-上期客户数量/上期客户数量

它是网站在争取新客户时获得成功部分的比例, 它反映了网站挖掘潜在市场、扩大市场 占有率的能力。同时, 网站开发新顾客的能力是网站提高市场份额的关键。

2.2.3 网站国际认知度

主观指标。指标数据获取方法: 问卷调查。

它是衡量消费者对网站品牌内涵及价值的认识和理解度的标准。B2B跨境电子商务网站 的国际认知度是网站在国际市场竞争力的一种体现, 有时会成为一种核心竞争力。

2.2.4 品牌忠诚度

客观指标。计算方式: 客户保持率=上期成交客户数在统计期间成交客户数/上期客户数 $* 100 \%$

品牌忠诚度最直观地表现在客户保持率上, 它是品牌价值的核心, 也是衡量网站社会效 益和竞争力的一个重要指标。企业留住老顾客的能力是企业保持市场份额的关键。

2.2.5 进出口贸易均衡性

客观指标。指标衡量方法: 进口贸易额与出口贸易额的比值。

跨境B2B电子商务网站在进出口贸易中应发挥积极的中间作用，应尽量减少贸易失衡问 题, 不仅要把控进出口贸易的质量, 也需要平衡好进出口贸易额度。充分吸引境内境外的商 家客户，匹配和对接双边供需信息，尽可能地缩小供需差距。

\section{3 服务与管理}

\subsection{1 个性化定制能力}

主观指标。指标数据获取: 问卷调查。

跨境B2B电子商务网站, 不同于传统的区域性B2B电子商务网站, “跨境”这一性质，会使 其面临更复杂的交易环境。网站能够根据不同的买家情况提供不同的个性化方案是该类网站 重要的能力。应考虑到国际市场客户层次结构和地域政策的不同, 提供不同的推荐方案, 合 同条款，支付选择、购买授权规则、审批流程、产品组合和营销策略。同时，基于不同地域 的文化背景, 网站呈现出不同的商品展示方式, 也显得十分重要, 比如多种语言环境下的动 态的推荐和搜索功能等, 允许买家只选择符合需求且适应多方法律政策的产品。

\subsection{2 外汇支付与结汇方式}

主观指标。指标衡量方式: 专家打分。

跨境电商作为一种新兴的贸易方式, 与传统贸易方式存在很大的区别, 主要表现在交易 和支付方式上, 跨境电商是以互联网为平台, 进行线上交易和支付。该网站是否支持跨境外 汇支付, 是否有稳定且合法的结汇渠道, 能否做到在短时间内支付和结汇, 以及如果支持第 三方支付平台支付，对于支付平台是否有相应的规范制度等都是重要的评判方向。

\subsection{3 交易安全性}

主观指标。指标衡量方法：第三方评估机构或专家打分。

支付安全方面：对网站选取的支付方式进行评估，评判方向可由网站的支付方式具体确 定, 分为直接支付和间接支付。而客户支付过程的风险主要来源于欺骗和盗用信息, 以及网 上木马病毒威胁, 该网站是否有相应的措施来规避或者减小支付风险是十分重要的评判标准。

交易保障方面: 评判该要素时应考虑网站是否采取了有效措施去监管供应方用户供应的 产品质量, 以及需求方需要退换商品时, 网站是否有保障供应方利益的措施。

2.3.4 信用管理

主观指标。指标数据来源：第三方评估机构。 
对于B2B跨境电子商务活动，用户的区域覆盖面广，不同的区域政治因素或者不同的商 业文化背景下, 用户会有不同的商业行为习惯, 而信用评级就显得尤为重要, 是保障B2B供 需双方利益, 和网站自身正常运营的一个重要参考指标。该网站是否有相关制度或借助第三 方信用评估机构对用户的信用等级进行测评, 并根据评估结果给予不同用户不同的权限, 显 得十分重要。

\subsection{5 信息系统建设}

主观指标。指标衡量方法: 专家打分。

跨境B2B电子商务网站贸易活动的开展必须依赖于良好的网站信息体统建设。客户的供 求信息，物流跟踪监管信息，库存信息，质量监管信息等应该被即时关注。

\subsection{6 跨境争端解决机制}

主观指标。指标衡量方法: 专家打分。

跨境B2B电子商务网站服务地区涉及文化和法律的差异, 网站在提供服务的过程中, 是 否提供了明确的法律声明, 是否有法律应急预案, 或者当不同国家的交易主体发生争端时, 网站是否能够较快地明确解决程序并推出解决方案等都是评判该指标时应该考虑的因素。

\subsection{7 跨境物流的成本和周期}

主观指标。指标衡量方法: 专家打分。

B2B跨境电子商务, 涉及到国际物流, 网站对于物流方案的选择应该被充分考虑。它直 接反映了该电子商务网站的物流体系和配套基础设施完善程度, 网站的物流配送方案主要分 为两个大类: (1) 供应商自行配货模式, 该方案使物流方式变得多元化, 但是也存在物流效 率得不到统一, 给规范化管理增加了难度, 评估的时候寻找其中的一个平衡点十分重要。（2） 网站提供配货方案, 在该模式下网站能否协调好与国际物流公的合作关系、网站是否有针对 不同地域的仓库管理以及通关清关方案是重要衡量标准。

\section{4 确定指标权重}

构造两两比较判断矩阵, 运用T.L.Saaty提出的“1-9标度方法”, 采用发放专家问卷的形式 获取判断矩阵, 并根据指标因素的重要性分析构造出判断比较矩阵。综合成功收回的7分专家 问卷结果，利用YAAHP软件获取模型计算结果并进行一致性微调，可以得到各项指标权重， 经检验均符合一致性要求, 得到如下指标权重表（表1）:

表1 指标权重表

\begin{tabular}{|c|c|c|c|c|c|}
\hline 一级指标 & 权重 & 二级指标 & 权重 & 二级指标 & 合成权重 \\
\hline \multirow{11}{*}{$\begin{array}{c}\text { 跨境B2B电子商 } \\
\text { 务网站绩效 }\end{array}$} & \multirow{11}{*}{1} & & & 资产周转率C11 & 0.4673 \\
\hline & & 资金与财务B1 & 0.6301 & 成本费用净利率C12 & 0.0474 \\
\hline & & \multirow{6}{*}{ 社会与市场B2 } & \multirow{6}{*}{0.1085} & 交易额环比增长率C13 & 0.1153 \\
\hline & & & & 市场份额C21 & 0.0561 \\
\hline & & & & 客户获得率C22 & 0.0178 \\
\hline & & & & 网站国际认知度 $\mathrm{C} 23$ & 0.0096 \\
\hline & & & & 网站品牌忠诚度C24 & 0.0165 \\
\hline & & & & 进出口贸易均衡性C25 & 0.0085 \\
\hline & & \multirow{3}{*}{ 服务与管理B3 } & \multirow{3}{*}{0.2614} & 个性化定制能力C 31 & 0.0180 \\
\hline & & & & 外汇支付与结汇方式C32 & 0.0743 \\
\hline & & & & 交易安全性C33 & 0.0645 \\
\hline
\end{tabular}




\begin{tabular}{lc}
\hline 信用管理C34 & 0.0555 \\
信息系统建设C35 & 0.0168 \\
跨境争端解决机制C36 & 0.0147 \\
跨境物流成本及周期C37 & 0.0177
\end{tabular}

通过对各个层次指标进行对比分析，可以得到B2B跨境电子商务网站绩效影响中最值得 关注的是网站的资金与财务情况, 从底层指标来看, 资金周转提高率、交易额环比增长率和 外汇支付与结汇方式的权重值分别位居前三, 另外, 网站交易的安全性、市场份额和信用管 理同样不容忽视。

\section{3. 评价方法与步骤}

基于模糊数学的隶属度理论把定性评价转化为定量评价, 对受到多种因素制约的绩效评 价体系做出一个总体的直观评价。本文将跨境B2B电子商务网站绩效评价指标划分为了三个 等级, 所以需要构建三级模糊综合评价体系。

3.1构建评价因素集、权重集和评语集

根据上文AHP层次分析模型结果分别建立因素集和权重集:

一级评价因素集: $\boldsymbol{F}_{1}=(B 1, B 2, B 3)$ 。二级评价因素集: $\boldsymbol{F}_{21}=(C 11, C 12, C 13)$,

$$
\boldsymbol{F}_{22}=(C 21, C 22, C 23, C 24, C 25), \boldsymbol{F}_{23}=(C 31, C 32, C 33, C 34, C 35, C 36, C 37) 。
$$

一级指标权重集: $\boldsymbol{W}_{1}=\left(w_{1}, w_{2}, w_{3}\right)=(0.6301,0.2614,0.1085)$ 。

二 级 指 标 权 重 集: $\boldsymbol{W}_{21}=\left(w_{211}, w_{212}, w_{213}\right)=(0.7418,0.0752,0.183)$, $\boldsymbol{W}_{22}=\left(w_{221}, w_{222}, w_{223}, w_{224}, w_{225}\right)=(0.5168,0.1644,0.0885,0.1518,0.0785)$

$\boldsymbol{W}_{23}=\left(w_{231}, w_{232}, w_{233}, w_{234}, w_{235}, w_{236}, w_{237}\right)=(0.0688,0.2842,0.2465,0.2124,0.0641,0.0563,0.0676)$ 评语集: $\boldsymbol{E}=\left(E_{1}, E_{2}, E_{3}, E_{4}, E_{5}\right)=$ (很好, 好, 一般, 差, 很差)。

\section{2 构建模糊评价矩阵}

构建第一级模糊评价矩阵：针对三个层次的评价指标, 采用自底向上的顺序, 先在三级 指标与评语集E之间构建一级模糊评价矩阵A1-A3。矩阵A1如表2所示, 同理可得A2, A3。

表2 一级模糊评价矩阵A1

\begin{tabular}{|l|l|l|l|l|l|}
\hline $\mathrm{B} 1$ & $\mathrm{E} 1$ & $\mathrm{E} 2$ & $\mathrm{E} 3$ & $\mathrm{E} 4$ & $\mathrm{E} 5$ \\
\hline $\mathrm{C} 11$ & $\mathrm{e}_{111}$ & $\mathrm{e}_{112}$ & $\mathrm{e}_{113}$ & $\mathrm{e}_{114}$ & $\mathrm{e}_{115}$ \\
\hline $\mathrm{C} 12$ & $\mathrm{e}_{121}$ & $\mathrm{e}_{122}$ & $\mathrm{e}_{123}$ & $\mathrm{e}_{124}$ & $\mathrm{e}_{125}$ \\
\hline $\mathrm{C} 13$ & $\mathrm{e}_{131}$ & $\mathrm{e}_{132}$ & $\mathrm{e}_{133}$ & $\mathrm{e}_{134}$ & $\mathrm{e}_{135}$ \\
\hline
\end{tabular}

构建第二级模糊评价矩阵。根据公式 $r_{n}=W_{n} \cdot A_{n}$, 将二级指标权重集与其对应的一级模 糊评价矩阵做乘积运算, 可以得到二级指标B1-B3的评价结果集, 分别对应为 $\boldsymbol{r}_{1}, \boldsymbol{r}_{2}, \boldsymbol{r}_{3}$ 。例如 将二级指标资金与财务的权重集 $\boldsymbol{W}_{21}=(0.7418,0.0752,0.183$ 与其对应的模糊评价矩阵A1做 以上运算，可得该指标的评价结果集 $\boldsymbol{r}_{1}=\left(r_{11}, r_{12}, r_{13}, r_{14}, r_{15}\right)$, 其余二级指标同理。

构建第三级模糊评价矩阵。第一级模糊评价矩阵 R由第二级评价结果集 $\boldsymbol{R}=\left[\begin{array}{lll}r_{1} & r_{2} & r_{3}\end{array}\right]^{T}$ 组成,再次根据结果集公式 $\boldsymbol{r}_{\boldsymbol{n}}=\boldsymbol{W}_{\boldsymbol{n}} \cdot \boldsymbol{A}_{\boldsymbol{n}}$, 可以得到一级指标跨境 $\mathrm{B} 2 \mathrm{~B}$ 电子商务网站绩效的评价 结果集 $\boldsymbol{r}_{\boldsymbol{0}}=\left(r_{01}, r_{02}, r_{03}, r_{04}, r_{05}\right)$ 。

3.3 确定评价结果集 
采 用 百 分 制, 并 由 加 权 平 均 法 确 定 绩 效 水 平
$\boldsymbol{M}$ 如表3, 本文将跨境B2B电子商务网站绩效水平分为四个级别“较好”“中等”“较差”“很差”。 表3 跨境B2B电子商务网站绩效水平等级评价表

\begin{tabular}{|l|l|l|l|l|}
\hline 绩效水平等级 & 较好 & 中等 & 较差 & 很差 \\
\hline 绩效得分 $\mathrm{M}$ & $\mathrm{M} \geqq 80$ & $80>\mathrm{M} \geqq 65$ & $65>\mathrm{M} \geqq 60$ & $\mathrm{M}<60$ \\
\hline
\end{tabular}

最后根据 $\mathrm{M}$ 分值所在区间, 可以得到跨境B2B电子商务网站绩效水平评价结果。

\section{4. 总结}

本文针对跨境B2B电子商务网站的特性，以评价网站绩效水平为最终目标，从资金、市 场和服务管理的角度分析总结出 3 个二级指标和 15 个具有特色的三级指标, 采用AHP层次分析 法与模糊综合评价法相结合的评价方法, 主要使用YAAHP软件整合分析专家问卷及评审数 据, 经过优化和调整, 最终构建了一个相对完整的跨境B2B电子商务网站绩效评价方法体系, 最终可以得到直观的绩效水平评价结果, 并可以根据不同指标的权重和得分, 从多个方面发 现网站绩效存在的问题, 有助于找出改进或提升绩效水平的着手点, 从而帮助网站向更好的 方向发展。

\section{References}

[1] Wei Rong. Research on The Performance Evaluation of B2C E-Commerce Website [D]. Shanghai: Wei Rong, 2016.

[2] Xu Dan. On the security of online payment [J]. Electronic World, 2012, (23): 1-1

[3] Tobey network "inventory: the five elements of the success of B2B e-commerce" 2015-02-12 $13: 46: 32$

[4] Yu Yunbao.Research on Performance Evaluation of Network Marketing of E-Commerce Enterprise [D]. LiaoNing: Yu Yunbao, 2014.

[5] Xiong Fumeng, Zhang Yi. "Internet plus" era of international trade a new direction for the development of cross-border electricity supplier of [J]. Business Economy, 2016, (475): 74-74

[6] Li Qian. Research on the financial difficulties of cross-border e-commerce in the new period [J]. Finance and Accounting for International Commrce, 2015, (12): 18-19

[7] Luo Hanyang. B2B e-commerce mode analysis and thinking [J]. Commercial Research, 2014, (299): 152-152 